\title{
REDESCRIC̣ÃO DE BITHORACOCHAETA STEIN (DIPTERA, MUSCIDAE) COM COMENTÁRIOS SOBRE SUA POSIC̣ÃO SISTEMÁTICA
}

\author{
Henrique César G. da Motta ${ }^{1,2}$ \\ Márcia Souto Couri ${ }^{1,3}$
}

\begin{abstract}
REDESCRIPTION OF BITHORACOCHAETA STEIN, WITH COMMENTS ON ITS SYSTEMATIC POSITION (DIPTERA, MUSCIDAE). Bithoracochaeta Stein is redescribed based on type material and good series of other species. The redescription includes characteres recently used in the modern classification of Muscidae. Coments on the position of the genus in Coenosiinae subfamily and illustration of proboscis, katepisternum and female terminalia are also presented.

KEY WORDS. Bithoracochaeta, Muscidae, Taxonomy
\end{abstract}

Bithoracochaeta foi descrito por STEIN (1911), como um antomí́deo da América do Sul. MALLOCH (1934) teceu comentários sobre esse gênero, destacando caracteres que segregavam Bithoracochaeta dos demais gêneros neotropicais de Coenosiinae. Albuquerque (1956) reforçou a inclusão do gênero entre os "Coenosiini" porém não sustentou esta posição; comentou que para a segregação das espécies seriam necessárias dissecções das genitálias. HENNIG (1965), PONT (1972) e CARVALHO (1989b) confirmaram a posição de Bithoracochaeta entre os Coenisiinae neotropicais, o último autor, considerando-o na tribo Coenosiini. CARVALHO et al. (1993) consideraram oito espécies válidas para o gênero.

Embora Bithoracochaeta possa ser facilmente segregado dos demais gêneros afins, uma descrição mais detalhada faz-se necessária, para sustentar a posição do gênero dentro da classificação moderna de Muscidae.

\section{MATERIAL E MÉTODOS}

A redescrição do gênero foi baseada em material-tipo e em boas séries reunidas de coleções das instituições relacionadas abaixo, seguidas do nome do responsável pelo empréstimo:

BMNH - Department of Entomology, The Natural History Museum, Londres, Inglaterra, Dr. Brian Pitkin.

DZUP - Coleção Entomológica Pe. Jesus S. Moure, Departamento de

1) Museu Nacional, Universidade Federal do Rio de Janeiro, 20940-040 Rio de Janeiro, Rio de Janeiro, Brasil.

2) Bolsista da CAPES.

3) Bolsista do CNPq. 
Zoologia, Universidade Federal do Paraná, Curitiba, Paraná, Brasil. Dr. Claudio José Barros de Carvalho.

MNRJ - Museu Nacional, Universidade Federal do Rio de Janeiro, Rio de Janeiro, Brasil.

SMT - Staatliches Museum für Tierkunde, Dresden, Alemanha. Dr. Uwe Kallweit.

USNM - Systematic Entomology Laboratory, U.S. Departament of Agriculture c/o USMN, Washington, Estados Unidos da América do Norte. Dr. Raymond Gagné.

ZMUC - Zoologisk Museum, Universitets Copenhagen, Copenhagen, Dinamarca. Dr. Verner Michelsen.

Foram dissecados a probóscide e o ovipositor de um exemplar fêmea de Bithoracochaeta leucoprocta (Wiedemann, 1830), espécie tipo do gênero. As estruturas foram colocadas em uma solução de hidróxido de potássio, em banhomaria, por 10 minutos, após foram lavadas em álcool $70 \%$, dissecadas e desenhadas em glicerina não hidratada. Ao término da análise, o material dissecado foi acondicionado em tubinho de vidro com glicerina no fundo e fixado no alfinete junto com o espécime.

A metodologia de CARVAlho (1989b) foi utilizada, incluindo a matriz de dados, com alterações nos estados de carateres de Bithoracochaeta que não coincidiam com este autor. As abreviaturas utilizadas são as mesmas de CARVALHO (1989a) acrescidas de: $\sin =$ sinonímia, coment $=$ comentários.

\section{RESULTADOS E DISCUSSÃO}

\section{Bithoracochaeta Stein, 1911}

Bithoracochaeta Stein, 1911: 177(desc.). - Stein, 1919:157(cat.). - Malloch, 1921:44(sin.). - Curran, 1928: 87(coment). - Curran, 1934: 395(chave). - Malloch, 1934: 175, 225 (desc., coment). Huckett, 1934: 57, 59, 60-61 (chave, coment., sin., desc.). - Séguy, 1937: 188, 189, 544 (chave, desc., sin.). - Albuquerque, 1956, 1-2: (coment., sin., desc.). - Hennig, 1965: 68 (coment.): Pont, 1972: 36 (cat., sin.). - Couri \& Lopes 1985: 593 (chave). - Carvalho 1989b: 631, 632, 633, 637 (coment). - Carvalho et al., 1993: II-113 (cat.).

Descrição macho. olhos nus; dicópticos; arista com cílios basais cerca do dobro da largura da arista na base; flagelo com ápice acuminado; cerdas ocelares fortes, longas e divergentes; cerdas verticais internas longas, fortes convergentes e retrovertidas; cerdas verticais externas pequenas e divergentes; cerdas pósverticais finas e convergentes; cerdas pós-oculares, pouco menores que as cerdas verticais externas; quatro pares de cerdas frontais longas; acrosticais pré-suturais desenvolvidas; cerda pré-alar ausente; notopleura sem cílios de revestimento; cerdas notopleurais de comprimento semelhante entre si; espiráculo anterior estreito e alongado; catepímero nu; duas cerdas pró-epimerais; duas cerdas pró-episternais; asas com veias nuas; tíbia III com cerdas longas nas faces anteroventral, anterodorsal e posterodorsal inseridas no terço médio, algumas espécies com uma cerda na face posteroventral; primeiro esternito nu; cerdas laterais nos tergitos II a $\mathrm{V}$ e discais no tergito $\mathrm{V}$. 
Fêmea. Cerda vertical interna longa, dirigida para trás e vertical externa curta dirigida para fora; cerdas interfrontais ausentes. Probóscide (Fig. 4) com arco posterior do fulcro com a ponta desenvolvida maior que o comprimento total, curvatura inferior do fulcro acentuada; haustelo nu, parcialmente esclerotinizado; labelo com dentes prestomais desenvolvidos, palpo com cerdas fortemente esclerotinizadas. Ovipositor longo (Figs 1 e 2). Microtríquias presentes em todo o ovipositor; cercos mais longos que o epiprocto e do mesmo tamanho do hipoprocto; tergitos VI, VII e VIII, em forma de âncora, o tergito VIII mais afilado que os demais, com uma dilatação no ápice. Esternitos Ví e VII em forma de bastão afilado com extremidade posterior dilatada; esternito VIII reduzido a duas pequenas placas, apresentando 2 cerdas cada um. Três espermatecas sub-ovaladas (Fig. 3).

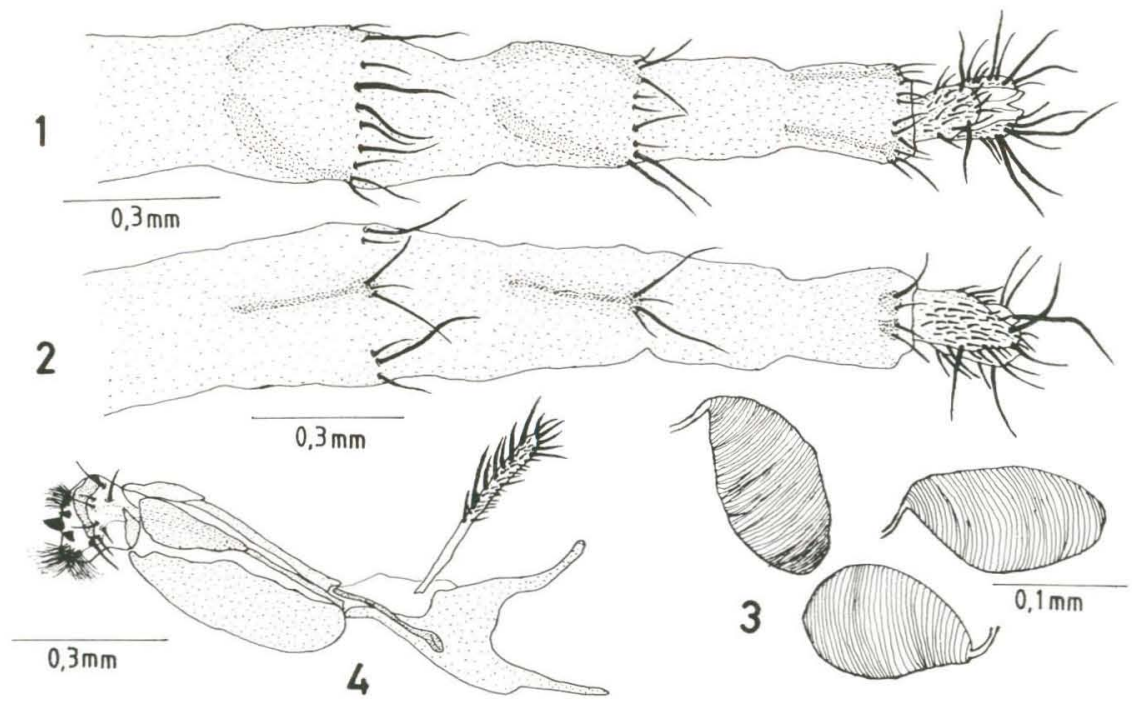

Figs 1-4. Bithoracochacta leucoprocta. (1) Ovipositor, vista dorsal: (2) ovipositor, vista ventral; (3) espermativas; (4) probóscide.

Espécie-tipo. Anthomyia despecta Walker, 1852 (des. orig.) =Bithoracochaeta leucoprocta (Wiedemann, 1830).

Comentários. Alguns estados de caráter discordam de CARVALHO (1989b), tais como: caráter 9: esternito VII inteiro; caráter 10: tergito VI esclerotinizado em forma de âncora; caráter 15: arco posterior do fulcro com a ponta desenvolvida maior que o comprimento total; caráter 32: as cerdas do catepisterno, embora tenham sido consideradas em triângulo equilátero, apresentam-se variáveis nas espécies (Figs 5-11).

Inserindo as alterações citadas acima na matriz de dados utilizada por Carvalho (1989b), foi confirmada a posição de Bithoracochaeta entre os Coenosiinae, Coenosiini, reforçada ainda pela presença da cerda pro-epimeral inferior curvada para baixo. 


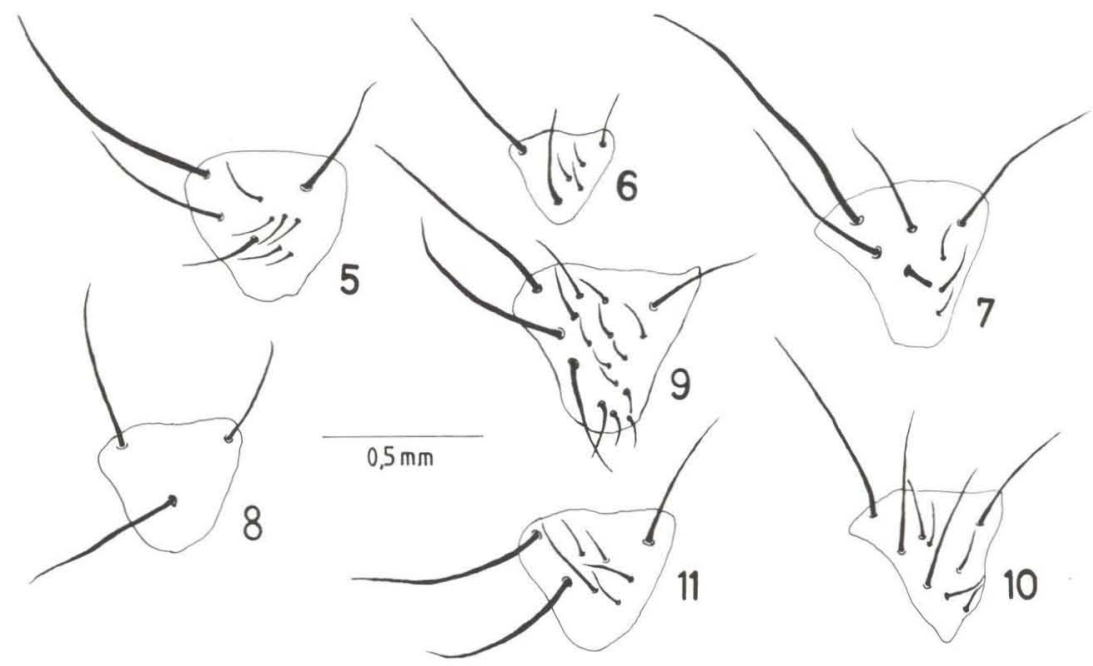

Figs 5-11. Catepisterno. (5) B. atricornis; (6) B. flavicoxa; (7) B. plumata; (8) B. nigricornis; (9) B. annulata; (10) B. varicornis; (11) B. leucoprocta.

Para lista de espécies válidas veja CARvalHo et al. (1993)

Material examinado. Bithoracochaeta annulata. PERU, Pachitea-Münd, Holótipo fêmea 20-XI-1903, W. Schnuse leg., (SMT). GuIANA IngLESA, Mazaruni, uma fêmea 27-VIII-1937, Richards e Smart leg. (BMNH). Suriname, Coronie, um macho 15-III-1945, Gersler leg. (MNRJ). BRASIL, Minas Gerais: Diamantina, 11 machos 13 fêmeas e dois de sexo não identificado 3-7-III-1956, Albuquerque leg. (MNRJ); Rio de Janeiro: Teresópolis, uma fềmea 25-I-1940, Lopes leg. (MNRJ). PERU, Laristhal, uma fêmea 14-VIII-1903, uma fêmea 15-VIII-1903, um macho 16-VIII-1903, W. Schnuse leg. (SMT). Rio Ucayali, uma fêmea 22-X-1903, W. Schnuse leg. (SMT).

Bithoracochaeta flavicoxa. CostA RiCA, S. Jose (Farm. La Caja), Parátipo macho e fêmea 15-IV-20-VI-1924 (BMNH); El SALVADOR, S. Tecla, uma fêmea e um de sexo não identificado 18-XII-1953, M. Salazar (USNM). CosTA RICA, San Jose (Farm La Caja), um macho e uma fêmea 15-IV-20-VI-1924 (BMNH); uma fêmea, J.F. Tristan leg. (USNM). GuiAnA INGLESA, Mazaruni, uma fêmea 13-VIII-1937, Richards \& Smart leg. (BMNH); sem localidae, uma fêmea 20-VIII-1929 (BMNH). BRASIL, Roraima: Ilha de Maracá (Rio Uraricoera), dois machos e quatro fêmeas 19-24-VII-1987, J. A. Rafael e L.S. Aquino leg. (DZUP).

Bithoracochaeta leucoprocta. WEST INDIANS, Lectótipo fêmea sem data (ZMUC). Est ados Unidos DA AMÉRICA, Florida: Fort Lauderdale, duas fêmeas 5-II-1950, O. Link leg. (USNM); De Soto (Arcadia), dois machos e uma fêmea 1-IV-1972, Miller e Kurczewski leg. (USNM); Orlando, um macho e uma fêmea 14-II-1918 (USNM); Key West, um macho 1-II-1969 (USNM); Sebring, um macho 20-III-1950, H.V. Weems Jr. leg. (USNM); Highlands Hamm St., um 
macho 20-III-1954, H.V. Weems Jr. leg. (USNM); Alachua, uma fêmea 29-XI-1953, F.W. Mead leg. (USNM); Alachua, uma fêmea 29-I-1954, H.V. Weems Jr. leg. (USNM); Bisc. Bay, um macho, Slosson leg. (USNM); St. Augustine, uma fêmea e um macho 18-IV-1919 (USNM); CH. Her, uma fềmea (USNM); Hialeah, um macho e uma fêmea 19-II-1967, C. Stegmaier leg. (USNM); Diaytim, um macho 3-IX-1894 (USNM); Royal Palm Park, um fềmea 22-IV-1930, A.L. Melander leg. (USNM); Lakeland, um macho e três fềmeas 6-V-1916, J.C. Bradley (USNM); Homestead, uma fêmea 18-II-1941, L.L. Pechuman leg. (USNM); Terra Ceia, uma fêmea 24-II-1946, J.G. Needham leg. (USNM); Sarasota, um de sexo não identificado, 21-II-1946, J.G. Needham leg. (USNM); Carolina do Norte: Southport, um macho 10-X-1948, C.W. Sabrosky leg. (USNM); Texas: Galveston, dois machos, duas fêmeas e um de sexo não identificado (USNM); Lousiana: Lake Charles, duas fềmeas 9-VI-1917, J.M. Aldrich leg. (USNM); Opelousas, dois macho e uma fềmea IV-1897, três machos V.1917, J.M. Aldrich leg. (USNM); New Orleans, um macho 29-III-1905, Jas S. Hine leg. (USNM); Mississipi: Pass Christian, um macho e uma fêmea 7-VI-1917, uma fêmea 8-VI-1917, J.M. Aldrich leg. (USNM); Georgia: Tifton, uma fếmea 30-VIII-1896, duas fềmeas 17-X-1896, A.L. Melander leg. (USNM). Okefenokee Swanp (Billy' Island), dois macho, uma fêmea e um de sexo não identificado, 15-VI-1912 (USNM). MÉxICO, Durango, dois machos e uma fêmea XI-1927, F.C. Bishopp leg. (USNM); Orizaha, um macho, uma fêmea e um de sexo não identificado 9-16-I-I892, H. Osborn leg. (USNM). CUBA, Havana, um macho e três fêmeas, Baker leg. (USNM); Cayanas, uma fềmea, Baker leg. (USNM). Costa Rica, San Jose, um de sexo não identificado, H. Schmidt leg. (USNM). PANAMÁ, Corazal (Canal Zone), uma fêmea 1-III-1972, Aug. Busk leg. (USNM). Brasil, Mincus Gerais: Diamantina, uma fêmea 3-7-III-1956, D. \& I. Albuquerque leg. (MNRJ); Nova Lima (Lagoa Grande), uma fêmea 13-II-1978, H.S. Lopes leg. (MNRJ). Rio de Janeiro: Teresópolis, quatro fêmeas XII-1939, duas fêmeas e um de sexo não identificado XI-1939, uma fêmea e dois de sexo não identificado XI-1940, duas fêmeas XI-1940, Freitas leg. (MNRJ); Itatiaia (Lago Azul), uma fêmea 16-18-1956, Rego Barros e D.O. Albuquerque leg. (MNRJ); Nova Friburgo, duas fêmeas 23-IV-1937, S. Lopes leg. (MNRJ); Petrópolis (Alto da Mosela), uma fêmea 6-II-1971, D.O. Albuquerque leg. (MNRJ); Vassouras, uma fêmea I-1940, D. Machado leg. (MNRJ); São Paulo: São José dos Campos, cinco fêmeas VII-1933, duas fềmeas X-1933, H.S. Lopes leg. (MNRJ); Campos do Jordão, uma fêmea 24-IX-1938, N. Santos (MNRJ); Sumaré, uma fêmea I-1942, Carrera leg. (MNRJ); Mogi das Cruzes, uma fêmea XI-1939, M. Carrera leg. (MNRJ); Itaquaquecetuba, uma fềmea 18-V, C.H.T. Townsend leg. (MNRJ); Paraná: Tijucas do Sul, dois machos e uma fềmea 23-24-XI-1979, Claudio Carvalho leg. (DZUP); Lapa, um macho 5-XII-1981, E.R. Silveira leg. (DZUP); Curitiba, uma fêmea 17-II-1966 (DZUP); Morretes (Vista Lacerda), uma fêmea 17-III-1966, Giacomel \& Marinoni leg. (DZUP); Santa Catarina: Seara (Nova Teutônia), um macho 16-VI-1937, uma fêmea 2-XI-1952, uma fêmea 30-XII-1934, uma fêmea 4-XI-1935, uma fêmea 5-XI-1935, um macho 5-XI-1936, uma fêmea 9-XI-1936, um macho 5-XII-1936, 
um macho 9-VI-1937, uma fêmea 15-VI-1937, um macho 2-VII-1937, uma fêmea 7-VII-1937, um de sexo não identificado 10-VI-1938, Fritz Plaumann leg. (BMNH); Rio Grande do Sul: Quaraí, um macho 21-XI-1985, J.R. Cure leg. (DZUFP). ARgEnTINA, Terr. Rio Negro: um macho 23-X-1926, F. \& M. Edwards leg., (BMNH) [Holótipo macho de Bithoracochaeta atricornis]. Jose C. Paz, um macho e duas fêmeas 24-VIII-1939, um macho e uma fềmea 25-VIII-1939, quatro fêmeas 2-X-1939, três fêmeas 10-IX-1939, Ogloblin (MNRJ); Buenos Aires (Vila del Parque), uma fềmea e um de sexo não identificado 13-VIII-1937, Ogloblin (MNRJ). Buenos Aires, três fềmeas 21-X-1926, F. \& M. Edwards (BMNH). UruguaI: Montevideo, um macho 21-22-I-1927, F\&M Edwards leg. (BMNH). ParaguaI: Sapucay, uma fềmea Fevereiro, W.T. Foster leg. (USNM).

Bithoracochaeta nigricornis. COSTA RICA, Higuito, San Mateo, holótipo fêmea, Pablo Schild leg. (USNM).

Bithoracochaeta plumata. BrasiL, Minas Gerais: Juiz de Fora, Salvaterra, holótipo macho I-1945, Lopes leg. (MNRJ); São Paulo, Onda Verde (Faz. São João), um macho I-1946, F. Lane leg. (MNRJ).

Bithoracochaeta varicornis. PORTO RICO, Viegues I., holótipo macho ( $\mathrm{n}^{\circ}$ 4375), parátipo macho $\left(n^{\circ} 4395\right)$ e parátipo fêmea $\left(n^{\circ} 4315\right)$ II-1899, Aug. Busck leg. (USNM). Brasil, Paraná: Dorado, uma fêmea 3-VII-1934 (MNRJ); Santa Catarina: Seara (Nova Teutônia), uma fềmea 15-XI-1936, Fritz Plaumann leg. (BMNH).

\section{REFERÊNCIAS BIBLIOGRÁFICAS}

Albuquerque, D.O. 1956. Contribuição ao conhecimento de Bithoracochaeta Stein, 1911 (Diptera-Muscidae). Bol. Mus. Nac. (n.s. Zool.), Rio de Janeiro, 149: $1-17$.

CARValho, C.J.B. DE. 1989a. Revisão das espécies e posição sistemática de Palpibracus Rondani (Diptera, Muscidae). Revta Bras. Zool. 6 (2): 325-375.

- 1989b. Muscidae (Diptera): uma proposta de análise cladística. Rev. Brasil. Biol. 44 (3): 335-337.

Carvalho, C.J.B.; A.C. Pont; M.S. Couri \& D. Pamplona. 1993. Part II. Muscidae. In: CARvalho, C.J.B. DE (ed.), A Catalogue of the Fanniidae and Muscidae (Diptera) of the Neotropical Region. São Paulo, Sociedade Brasileira de Entomologia.

COURI, M.S. \& S.M. LOPES. 1985. Neotropical Genera of Coenosiinae - Nomeclatural Notes and Key to identification (Diptera-Muscidae). Rev. Brasil. Biol. 45 (4): 585-595.

Curran, C.H. 1928. Diptera two winged tlies. Scient. Surv. Porto Rico \& Virgini. Is. New York 11: 1-118.

1934. The Families and Genera of North American Diptera. New

York, The Ballou Press, 512p.

HENNIG, W. 1965. Vorarbeiten zu einem phylogenetischen System der Muscidae

(Diptera: Cyclorrhapha). Stuttg. Beitr. Naturk. 141: 1-100. 
HucketT, H.C. 1934. A revision of the North American species belonging to the genus Coenosia Meigen and related genera. (Diptera: Muscidae) Part I. The subgenera Neodexiopsis, Coenosia, Hoplogaster and related genera Allognota, Bithoracochaeta and Schoenomyza. Trans Amer. Ent. Soc. 60: 57-119.

MALLOCH, J.R. 1921. Notes on some of van der Wulp' species of north American. Anthomyidae (Diptera). Ent. News 32: 40-45.

- 1934. Diptera of Patagonia and South Chile. Muscidae. London, Part 7 (2), p. 171-346.

Pont, A.C. 1972. A Catalogue of the Diptera of the Americas South of the United States. 97. Family Muscidae. São Paulo, Museu de Zoologia, Universidade de São Paulo, $111 \mathrm{p}$.

SÉGUY, E. 1937. In: P. WYTSMAN (ed.). Genera Insectorum. 205. Fam. Muscidae, p. 1-604.

STEIN, P. 1911. Die von Schnuse in Südamerika gefangenen Anthomyiden. Arch. Naturg., Berlin, 77: 61-169.

1919. Die Anthomyidengattungen der Welt, analytisch bearbeitet, nebst einem kritisch-systematischen Verzeichnis aller aussereuropäischen Arten. Arch. Naturg., Berlin, 83A (1): 85-178.

Recebido em 30.V.1994; aceito em 13.II.1995. 